\title{
A survey of reproductive parameters in the free-ranging chimpanzees of Gombe National Park
}

\author{
J. Wallis* \\ Department of Obstetrics and Gynecology, University of Oklahoma Health Sciences Center, \\ Oklahoma City, OK, USA
}

\begin{abstract}
This study is a review of reproductive records for chimpanzees (Pan troglodytes schweinfurthii) living in Gombe National Park, Tanzania, between 1964 and 1994. Females exhibited their first full anogenital swelling at approximately 10.8 years of age. Duration of adolescent infertility averaged 2.37 years, with first conception occurring at 12.7 years and first parturition at 13.3 years. The menstrual cycles of young nulliparous females were significantly longer than those of older multiparous females (average 39.8 days versus 33.8 days). Analysis of anogenital swelling patterns indicated an average duration of 12-13 days for maximal swelling in all classes of cycle. Nulliparous and postpartum females exhibited a similar pattern of shortened detumescent phase, indicative of anovulation. Duration of gestation averaged 225.3 days (range $=208-235$ ) with no significant fetal gender effect. When an infant lived to weaning age, the postpartum amenorrhoea of the mother averaged 3.86 years, whereas if the offspring died as an infant the mother resumed menstrual cycles an average of 35 days later. Once postpartum cycles resumed, the next conception averaged 142.5 days later if the previous infant had survived, but sooner (92.9 days later) if the previous offspring died in infancy. Interbirth interval averaged 5.15 years. Analysis of behavioural records indicated that a lower percentage of conceptions occurred during consort matings than was previously thought, suggesting additional research is needed on this topic. Detailed discussion focuses on the environmental influence on reproductive parameters described here. Future work in this area will probably identify the importance of diet - via basic nutrition and phytochemical content - in effecting the seasonal and individual differences seen in this study.
\end{abstract}

\section{Introduction}

The study of chimpanzee reproduction has a long and successful history. The earliest scientific investigations of laboratory chimpanzees (in the 1930s) addressed such issues as menstrual cycle and pregnancy duration as well as other reproductive parameters. Because those studies were conducted in a captive setting, the findings were generally given in exact figures and were supported with hormonal measurement to examine, among other aspects, the proximate cause of the periodic anogenital swelling occurring in menstrual cycles of female chimpanzees (Yerkes and Elder, 1936; 1937; Young and Yerkes, 1943).

It was not until the late 1960 s that field researchers could address reproductive concerns in wild chimpanzees. Owing to longevity and slow maturation rate of chimpanzees, in addition to the difficulties of reliably observing subjects in the field, most reports concerning reproductive parameters under natural conditions rely heavily on estimates of age. These often provide only scant data from small sample sizes, and as yet are

*Current address: Oklahoma Biological Survey, University of Oklahoma, 111 E. Chesapeake Street, Norman, Oklahoma, 73019-0575, USA.

Received 26 June 1996 not accompanied by hormonal data. However, field studies have been extremely valuable in aiding our understanding of chimpanzee biology, particularly as they confirm the importance of environmental influence as indicated by many differences between captive and wild populations. These differences are almost entirely attributable to nutrition. Elder and Yerkes (1936) first noted that '... good diet, social and hygienic conditions are necessary for reproduction to proceed naturally ... citing nutritional inadequacy or emotional disturbance as the primary cause of spontaneous abortions in chimpanzees.

\section{Chimpanzee reproductive cycles and anogenital swellings}

As puberty approaches, the genital skin of a female chimpanzee begins to show variable swelling in response to increasing concentrations of circulating steroids. The swelling is due to an organ-specific, oestrogen-induced effect on hyaluric acid, resulting in water accumulation (see Graham, 1981). Chimpanzees exhibit a long period of adolescent 'sterility' (or, more accurately, 'infertility') from the time of menarche to the first conception. Young and Yerkes (1943) suggested that, in adolescence, ovulation may occur but the secretion of progesterone is typically not adequate to support pregnancy, 
that is, the failure is one of luteal function rather than of ovulation.

Once maturity is reached, menstrual cycles become more regular and the hormonally controlled swelling involves skin surrounding the entire anogenital region. This anogenital swelling, often referred to as 'sexual swelling' or 'oestrous swelling', fluctuates cyclically, gradually increasing during the follicular phase and staying at maximal turgidity during the peak concentration of oestrogen. With the increase of progesterone, there is a rather sharp decrease in swelling and total quiescence lasts until menstruation occurs and the menstrual cycle begins again. Ovulation typically occurs on or around the last day of maximal tumescence (see Graham, 1981; Nadler et al., 1985; Steinetz et al., 1992, for review of chimpanzee reproductive endocrinology).

The measurement of anogenital swelling, and the charting thereof, has long been used by researchers to track reproductive health, identify pregnancy, and predict parturition dates. Modern assessment of chimpanzee anogenital swelling separates the gradual swelling (termed tumescence) seen early in the follicular phase from that of maximal tumescence. The partial swelling that occurs in the early luteal phase is termed detumescence. Another method of monitoring chimpanzee reproductive cycles cites labial occlusion as having the potential to predict time of ovulation and to assess the adequacy of the cycle (Dahl et al., 1991).

Anogenital swelling - and its correlation with sexual activity - continues sporadically during pregnancy of both captive (Tinklepaugh, 1933; Yerkes and Elder, 1937; Wallis, 1982; Wallis and Lemmon, 1986) and wild (van LawickGoodall, 1969; Tutin and McGinnis, 1981; Wallis and Goodall, 1993; Wallis and Bettinger, 1993) chimpanzees. The hormonal mechanism producing this swelling is not well understood, but gestation-related anogenital swelling occurs most often in early pregnancy and is negatively correlated with the age of the mother (captive: Wallis and Lemmon, 1986; wild: Wallis and Goodall, 1993).

Occasional anogenital swellings may also occur during lactation. These swellings are more erratic and less frequent than those occurring in pregnancy, but are still accompanied by sexual interest from males (Wallis and Bettinger, 1993). Moreover, a female with an infant may exhibit a 'false start' swelling cycle which appears normal, but occurs significantly earlier than expected and is followed by several months or even a year of anogenital quiescence before regular menstrual cycles begin (Wallis, 1992b). False start cycles are not seen in females after infant loss; those mothers typically resume cycles very quickly. However, most female chimpanzees are still nursing an infant when they resume postpartum cycles and weaning may not be complete until the mother is already pregnant again (Tutin and McGinnis, 1981). Therefore, the term 'postpartum amenorrhoea' is a more accurate term than 'lactational amenorrhoea' for chimpanzees.

\section{Consortships}

Three distinct mating patterns have been described for chimpanzees (McGinnis, 1979; Tutin, 1979; 1980; Hasegawa and Hiraiwa-Hasegawa, 1983; and see review in Morin, 1993).
Chimpanzee copulations may occur opportunistically, within the group, in which a female may be mated by all males of the community. Alternatively, they may occur in more restrictive patterns, either within the group, where a high-ranking male prevents other males from mating with a female (possessive mating), or away from the group, where a male escorts a female away for exclusive copulatory access (consortships). Tutin (1979) reported that $73 \%$ of copulations were in opportunistic, $25 \%$ possessive, and only $2 \%$ occurred in consort settings. However, by definition, consort partners try to avoid contact with others (including researchers); therefore, it is difficult to obtain an accurate assessment of these mating patterns.

\section{Seasonal influence on reproduction}

Young and Yerkes (1943) cited evidence of seasonal amenorrhea in captive chimpanzees: the duration of menstrual cycles increased during North American winter months. In addition, they found that menarche tended to occur most often during the summer months, which was also the most favourable time for normal cycle activity.

A strong seasonal influence is seen in a number of reproductive aspects of wild chimpanzees. Females resume postpartum cycles most often in the dry season (Goodall, 1986; Nishida et al., 1990; Wallis, 1992b; 1995). In addition, the first full anogenital swelling in young females, anogenital swelling in pregnant and lactating females, maximal swelling in cyclic females, and the timing of conception are all seasonally influenced, each reaching peaks in the late dry season (August to October) (Wallis, 1995). Seasonal variation in diet is cited as the possible cause of this phenomenon.

As indicated above, most reports of reproduction in wild chimpanzees have relied heavily on estimates or contain very small sample sizes. The present study, a survey of the long-term records of free-ranging chimpanzees in Gombe National Park, supplements those earlier reports. The objectives were to re-examine and expand the previous findings, address aspects of the reproductive profiles of this population that have not been discussed previously, and suggest potential areas for future investigation. Specifically related to the three sections above, this study: (1) assesses anogenital swelling patterns as they relate to age, parity, and fertility; (2) investigates the incidence of conceptions resulting during consortships; and (3) examines the seasonal influence on the duration of reproductive cycles of chimpanzees.

\section{Methods}

The study subjects were free-ranging chimpanzees (Pan troglodytes schweinfurthii) living in Gombe National Park, Tanzania, East Africa. Gombe is located on the eastern shore of Lake Tanganyika at $4^{\circ} 40^{\prime} \mathrm{S}, 29^{\circ} 38^{\prime} \mathrm{E}$. It consists of a 10 mile by 3 mile strip of mountainous terrain, composed of alternating thick riverine forests, deciduous woodland, and hilltop grassland (see Goodall, 1986, for a detailed history of Gombe). The study population, the Kasakela Community, ranges in the central portion of the Park, and has consisted of approximately 40-45 chimpanzees throughout most of the period under review. 
For assessment of general issues such as timing of reproductive events, infant gender and infant survival rate, the data set included all records from January 1964 to December 1994. However, for detailed analysis of duration of gestation, duration of menstrual cycle, and anogenital swelling patterns, only data from mid-1975 to mid-1994 were used, as these are the most reliable records available. Because Gombe data collection has focussed more on male subjects, and because female chimpanzees tend to exercise a great deal of variability in their travel patterns and social contact (i.e., they are not observed every day), only portions of the data on reproductive parameters were judged adequate for analysis.

\section{Duration of menstrual cycle}

The duration of menstrual cycles of free-ranging chimpanzees can be difficult to assess because females are often absent (on consortship or travelling near the community border) during part of the oestrous phase. Therefore, only a selected number of cycles was considered adequate for this study. Traditional menstrual cycle assessment designates day 1 as the first day of observed menstrual flow (or the first day after menstrual flow has ended). However, because menstrual blood is often not detected under free-ranging conditions, duration of menstrual cycle for this analysis was measured from the day after maximal anogenital swelling ended in one cycle to the last day of maximal swelling in the following cycle. Cycle duration was determined for three classes: cycles of nulliparous females, and infertile and fertile cycles of multiparous females. (A fertile cycle was defined as a cycle resulting in conception as indicated by subsequent evidence of miscarriage, stillbirth, or live birth.) A Student's $t$ test was used to determine differences between groups.

\section{Anogenital swelling phases}

At Gombe, a five-stage scale is used to measure the degree of anogenital swelling in female chimpanzees: $0=$ no swelling; $I=$ maximal tumescence; and $1 / 4,1 / 2,3 / 4=$ fractions thereof. For the anogenital swelling phase assessment of this study, the three fractions were combined to represent a partial swelling level. For this analysis, the partial swelling phase preceding maximal tumescence was termed 'tumescence' and the partial swelling phase following maximal tumescence was termed 'detumescence'.

The duration of anogenital swelling phases was determined for five reproductive classes. For the multiparous females, cycles were grouped according to whether they were a first postpartum cycle (i.e. a cycle preceded by postpartum amenorrhoea) or a 'regular' cycle (i.e. a cycle preceded by another cycle). Each of these was further subdivided into fertile and infertile classes. In addition, anogenital swelling phases were calculated for post-menarcheal nulliparous females. A Student's $t$ test was used to determine differences between groups.

Because free-ranging subjects are not reliably observed every day, the database included some cycles for which data for some days were missing. A conservative method of assessment was used for the missing days, i.e. if a female was partially swollen, followed by a day of missing data, and then a day of maximal anogenital swelling, the missing day was assumed to be a partial (not full) swelling day. This analysis used only cycles for which there was a minimal number of missing data.

Erratic and unusual anogenital swellings may occur during chimpanzee pregnancy (Yerkes and Elder, 1937; van LawickGoodall, 1969; Wallis and Lemmon, 1986; Wallis and Goodall, 1993); often the anogenital skin of a young pregnant female detumesces only slightly after conception and remains in a partially swollen state for several weeks. Therefore, the detumescent phase of fertile cycles was not assessed for comparison in this study.

\section{Age at first full anogenital swelling}

For this analysis, data were considered for only the young females of precisely known age using the criteria for their birthdate as described below. Because of the irregular nature of early anogenital swelling patterns for adolescent females, the occurrence of the first full anogenital swelling of a female chimpanzee does not always indicate normal cyclicity; the first full swelling may last only 3 or 4 days (Wallis, 1994).

\section{Estimate of gestation duration, conception and parturition}

For analysis of gestation duration, no pregnancies were included in which the first record of an infant gave an estimate of more than 3 days of age. In fact, most cases used in this study involved infants identified on the first day of life, that is, the mother was seen with a newborn 1 day after having been observed as still pregnant (and with no infant). In addition, when a retrospective review was made for the estimated time of conception, if the female was not seen for several days during the periovulatory period, that pregnancy was excluded from the duration estimate. For simplicity, the last day of maximal anogenital swelling during the last normal swelling cycle (the fertile cycle) was considered the day of conception (Graham, 1981). All stillbirths were omitted from the gestation duration analysis as it was impossible to determine whether death was due to prematurity (shortened gestation) or another cause. In addition, because multiple births tend to be premature (humans: Jones et al,, 1990; chimpanzees: Yerkes, 1934), the gestation duration of the one twin birth is provided separately but was omitted from the analysis.

Once the dates were determined for first anogenital swelling, conception, parturition and resumption of postpartum cycles, calculations were made for: adolescent infertility, estimate of duration of gestation, interbirth interval, duration of postpartum amenorrhoea, and time from resumption of postpartum cycles to the next conception.

\section{Consortships}

A review of the Gombe chimpanzee behavioural records from May 1975 to April 1994 identified conceptions that may have occurred during a consortship. As with previous references on this subject (McGinnis, 1979; Tutin, 1979; Tutin and McGinnis, 1981), consort pairings were often inferred from the records; when an adult male was not travelling with the main 
Table 1. Average duration of menstrual cycle and anogenital swelling patterns in free-ranging chimpanzees of Gombe National Park

\begin{tabular}{|c|c|c|c|c|c|}
\hline Parameter & $\begin{array}{l}\text { Regular cycles of } \\
\text { nulliparous females } \\
\text { (Infertile) } \\
(n=20)\end{array}$ & $\begin{array}{l}\text { Regular cycles of } \\
\text { multiparous females } \\
\text { (Infertile) } \\
(n=33)\end{array}$ & $\begin{array}{c}\text { Regular cycles of } \\
\text { multiparous females } \\
(\text { Fertile }) \\
(n=20)^{*^{+}}\end{array}$ & $\begin{array}{l}\text { First postpartum } \\
\text { cycles } \\
\text { (Fertile) } \\
(n=4)^{\dagger}\end{array}$ & $\begin{array}{l}\text { First postpartum } \\
\text { cycles } \\
\text { (Infertile) } \\
(n=16)\end{array}$ \\
\hline \multicolumn{6}{|l|}{ Menstrual cycle (in days): } \\
\hline Cycle duration & 39.8 & 33.8 & 33.2 & - & - \\
\hline Cycle range & $18-64$ & $27-56$ & $26-50$ & - & - \\
\hline \multicolumn{6}{|c|}{ Anogenital swelling phases (in days): } \\
\hline Tumescence & 4.6 & 4.2 & 2.5 & 7.5 & 6.3 \\
\hline Maximal tumescence & 12.5 & 12.3 & 13.1 & 12.5 & 13.2 \\
\hline Detumescence & 2.2 & 3.5 & - & - & 1.8 \\
\hline Total duration of swelling & 19.1 & 20.2 & - & - & 20.6 \\
\hline
\end{tabular}

*For this group, swelling phase data were from 17 cycles only.

${ }^{\dagger}$ For fertile cycles, no data for detumesence are provided.

group of adult males and an adult female was also absent from the community during the same period, this was considered an implied consortship. Very often, however, the consorting pair was observed by research staff and a 'consortship' was recorded as confirmed. Post-hoc analysis of the records determined whether the female was cyclic, acyclic (in postpartum amenorrhoea), or pregnant at the time of consortship regardless of the specific anogenital swelling present.

\section{Assessment of seasonality}

For determination of seasonal influence, the data were divided into seasonal quarters, based on long-term rainfall patterns at Gombe National Park (see Wallis, 1995). The quarters were: early dry season=May-July; late dry season $=$ August - October; early wet season $=$ November January; and late wet season = February-April. A general linear model was used to assess seasonal quarter effect.

\section{Results}

\section{Duration of menstrual cycles}

Analysis of duration of menstrual cycles was made on the following data set: cycles of nulliparous females -20 cycles from six subjects; multiparous infertile cycles -33 cycles from six subjects; multiparous fertile cycles -20 cycles from 14 subjects.

If the infertile, regular cycles exhibited by the nulliparous and multiparous females are considered together, the overall duration of menstrual cycles for Gombe chimpanzees was 36.1 days (range 18-64 days; $n=53$ ). However, when the reproductive histories of the females were considered, cycles of young nulliparous females were found to be significantly longer than those of multiparous females (39.8 days versus 33.8 days, $t=-2.06, P<0.05$ ). For multiparous females, there was no significant difference in duration between fertile and infertile cycles (Table 1).

\section{Anogenital swelling phases}

For determination of anogenital swelling phases (Table 1), the following data sets were used: cycles of nulliparous females - 20 cycles from six females; regular multiparous cycles (infertile) - 33 cycles from six females; regular multiparous cycles (fertile) - 17 cycles from 11 females; infertile postpartum cycles - 17 cycles from eight females; and fertile postpartum cycles - four cycles from four females.

Comparison of the anogenital swelling patterns of nulliparous females with those of multiparous, infertile cycles showed a significant difference in the detumescent phase; nulliparous females showed a pattern of more rapid decrease in anogenital swelling after the last day of maximal tumescence $(t=2.09, P<0.04)$. Likewise, for infertile cycles of multiparous females, there was a significantly shorter detumescent phase of the first postpartum cycles compared with the subsequent cycles $(t=2.13, P<0.04)$. The tumescent phases of these classes showed no significant difference.

Although no detumescent assessment was made for the fertile cycles, there was variation in tumescence. Regular fertile cycles had a significantly shorter tumescent phase than did the infertile regular cycles $(t=-2.17, P<0.03)$. In an assessment of fertile cycles only, the first postpartum cycles had a longer tumescent phase than the regular cycles $(t=-3.21$, $P<0.005$ ). An analysis of all postpartum cycles, comparing fertile with infertile, indicated no significant difference in tumescent nor maximal tumescent phases. Similarly, there were no significant differences in any of the swelling categories in comparison of nulliparous females' cycles and first postpartum cycles.

\section{Major reproductive parameters}

Reproductive milestones of young females. Eight young female chimpanzees of exact known age exhibited their first full anogenital swelling during the period under review (Table 2). Observation of four of these yielded sufficient data for assessing timing of the first pregnancy. The average age of 
Table 2. Reproductive parameters of the free-ranging chimpanzees of Gombe National Park

\begin{tabular}{lrrr}
\hline Parameter & $n$ & Mean & Range \\
\hline $\begin{array}{l}\text { Age at first full anogenital swelling } \\
\text { Adolescent infertility - duration }\end{array}$ & 8 & 10.8 years & $8.5-13.5$ years \\
Age at first conception & 4 & 2.4 years & $0.65-4.9$ years \\
Age at first parturition & 4 & 12.7 years & $10.5-16.6$ years \\
$\begin{array}{l}\text { Duration of gestation } \\
\text { 'Postpartum amenorrhoea' }\end{array}$ & 4 & 13.3 years & $11.1-17.2$ years \\
$\quad$ After infant lived & 28 & 225.3 days & $208-235$ days \\
$\quad$ (from parturition until cycle resumption) & 12 & 3.86 years & $2.41-5.69$ years \\
$\quad \begin{array}{l}\text { After infant died } \\
\quad \text { from infant death until cycle resumption) }\end{array}$ & 4 & 35 days & $14-77$ days \\
$\begin{array}{l}\text { Postpartum days to conception } \\
\quad \text { After previous infant lived }\end{array}$ & & & $16-402$ days \\
$\quad$ After previous infant died & & 17 & $24-152$ days \\
Interbirth interval & 9 & 92.9 days & $3.26-7.78$ years \\
\hline
\end{tabular}

first full swelling, first conception, first parturition, and the duration of adolescent infertility (the time between onset of first full swelling and the date of first conception) are shown (Table 2).

Duration of gestation. Only a small number of cases qualified for inclusion in the analysis of duration of gestation, owing to infrequent observation of the subjects or incomplete data collection. Duration of gestation averaged 225.3 days $(n=28$; range $=208-235$; Table 2 ), with no significant effect of fetal gender and no effect of (estimated) age of mother. One twin birth, not included in this analysis, occurred after 206 days of gestation.

Postpartum amenorrhoea. When an infant lived to weaning age, the postpartum amenorrhoea of the mother lasted an average of 3.86 years, whereas if the offspring died as an infant the mother resumed reproductive cycles an average of 35 days after infant death $(F=85.36, P<0.0001)$.

Number of postpartum days to conception. After resumption of postpartum menstrual cycles, the next conception occurred an average of 142.5 days later if the previous infant had survived and an average of 92.9 days later if the previous offspring died in infancy. This difference was not quite significant $(t=-1.42, P<0.08)$.

Interbirth interval. For 11 cases (from six females), the interbirth interval was on average 5.15 years (Table 2) with no significant gender effect. When all 11 cases were analysed together, there was no correlation between interbirth interval and age of the mother (using birthyear estimates on record) $(r=-0.007 ; P=0.98)$. However, one female, Fifi, contributed five of the samples used in this analysis. When only her data were considered, there was a significant negative correlation between interbirth interval and age $(r=-0.912 ; P=0.03)$. The duration of five successive interbirth intervals was 5.II, $4.72,3.89,4.27$ and 3.26 years.

\section{Gender and survival of infants}

Of 91 pregnancies occurring in 1964-1994, infant gender could be determined for 82 . There were 37 female and 45 male infants (actually, 46, as one pregnancy resulted in twins). Of these, $75.8 \%$ of the females and $65.9 \%$ of the males lived to juvenile age.

\section{Consortships}

A review of the census charts for May 1975 to April 1994 indicated 117 implied consortships. Fourteen of these represent seven occasions on which two females were consorting with one male at the same time. From the conception assessment data (described above), the 117 consort pairings yielded 14 (11.9\%) conceptions. These 14 consort conceptions represent $25 \%$ of the 56 known-month conceptions occurring during the 19 year period under review.

Although most of the 117 consort pairings included cyclic females, on ten occasions the females were already pregnant and on six occasions the females were acyclic and lactating, and had not resumed postpartum cycles. The duration of consortships ranged from 3 days to 3 months.

\section{Seasonality}

Of the postpartum cycles examined in this study, four were fertile, that is, conception occurred in the one and only cycle exhibited between successive deliveries. All four of these fertile postpartum cycles began during the month of June, resulting in conceptions in late June or July. (In addition to these four, there were two other postpartum cycles during the study period that led to conception but were not used in the analysis owing to lack of sufficient data. The onset of these, too, was in the dry season, one in July, the other in August.)

There was a seasonal effect on duration of infertile cycles of multiparous females. Cycles were shortest in the early dry season and longest in the late dry season $(F=3.27 ; P<0.04$; Table 3). Although the trend was similar for nulliparous 
Table 3. Average duration of menstrual cycle of Gombe chimpanzees assessed by seasonal quarter

\begin{tabular}{lcc}
\hline Seasonal quarter & $\begin{array}{c}\text { Nulliparous cycles } \\
\text { (Infertile; } n=20)\end{array}$ & $\begin{array}{c}\text { Multiparous cycles } \\
\text { (Infertile; } n=33 \text { ) }\end{array}$ \\
\hline Early dry (May-Jul.) & 39.0 days & 29.8 days \\
Late dry (Aug.-Oct.) & 41.6 days & 38.7 days \\
Eary wet (Nov.-Jan.) & 39.4 days & 32.4 days \\
Late wet (Feb.-Apr.) & 39.2 days & 31.9 days \\
ANOVA & $F=0.04$ & $F=3.27$ \\
(Seasonal effect) & (ns) & $(P<0.04)$ \\
\hline
\end{tabular}

ns: not significant.

females, the difference was not significant. The latter result should be taken as preliminary as the sample size $(n=20)$ may not be sufficiently large for quarterly analysis.

\section{Discussion}

It has become standard practice to refer to chimpanzee menstrual cycles as lasting approximately 36 days (33-36 days, Tinklepaugh, 1933; 36.2 days, Yerkes and Elder, 1936; 36.7 days, Wallis, 1992a). However, the duration of the cycle varies with age although no proper investigation of this issue has been made until now. Young and Yerkes (1943) first noted that young nulliparous females exhibit longer cycles than do older females. Although they analysed 653 cycles for their report, and provided a mean of 37.28 days, they did not offer age- or parity-specific values to support their claim that cycle duration decreases with age. Similarly, Tutin and McGinnis (1981) reported an average cycle duration of 36 days (range 25-84) for nine females at Gombe, but although they identified three subjects as nulliparous and six as multiparous, they did not give details of cycle duration by age class. van Lawick-Goodall (1969) referred to a median cycle duration of 41 days for six young females, but did not provide comparative data for older females. Likewise, a report from Mahale gave a cycle duration of 27-39 days, but did not provide the parity or age of the females (Nishida et al., 1990).

Although the present study confirmed that the overall average cycle duration is approximately 36 days - as cited in the literature - the reproductive history of the female must be considered when assessing menstrual cycle duration accurately. Confirming earlier implications from study of laboratoryhoused chimpanzees (Young and Yerkes, 1943), young nulliparous females at Gombe exhibited significantly longer menstrual cycles than did older multiparous females. The range of cycle duration was quite large; the difference between the minimum and maximum figures found in the present study (46 days) is greater than the average cycle duration (36 days).

Research on captive chimpanzees found that anogenital tumescence lasts for approximately 3-7 days, maximal tumescence 10-12 days, and detumescence 5 days (Yerkes and Elder, 1936; Graham, 1970; Wallis, 1992a). However, these studies did not separate the data by age class or fertility status; perhaps future research in captive settings will investigate these patterns in more detail.

The most often quoted data from field studies gives a slightly misleading picture; McGinnis (1979) provided information on the average anogenital swelling phases for seven females, implying that these figures were standard for wild chimpanzees: no swelling -13.6 days; tumescence -7.6 days; maximal tumescence - 16.3 days; and detumesence -4.5 days. As can be seen, these average figures equal a 42 day cycle, and a footnote in that publication identified all subjects as nulliparous. Because young females have longer cycles than do older females and tend to have a shortened postswelling period - suggesting a short and inadequate luteal phase (Young and Yerkes, 1943), caution must be used in applying these figures to a general population. This problem was addressed in the present study using a more detailed examination of the Gombe chimpanzee records and assessing anogenital swelling phases in several classes of cycle.

In all classes analysed, the average duration of maximal anogenital swelling remained the same; maximal anogenital swelling in chimpanzees lasts approximately 12-13 days. However, there was variation, in the time required for the anogenital swelling to reach maximal tumescence (during 'tumescence') and variation in the time taken for the anogenital swelling to decrease from maximal to total quiescence (during 'detumescence').

There was a significantly shorter detumescent phase for nulliparous females than for their multiparous peers. This pattern, well documented in previous research, suggests anovulation in cycles of young females (Young and Yerkes, 1943; Clark and Birch, 1948; Graham, 1970).

As observed by Young and Yerkes (1943), the postpartum cycles reverted to the adolescent pattern of a prolonged tumescent phase and shortened detumescent phase. These patterns are indicative of inadequate progesterone and oestrogen production, again indicating possibly anovulatory cycles (Nadler et al., 1981). However, even the fertile postpartum cycles showed a long tumescent phase in the present study. Young and Yerkes suggested that, after pregnancy, there may be a short period of (re)adjustment in the pituitary comparable to the maturation process in adolescence. It may take more time for the reproductive system (and anogenital skin) to resume a pattern of swelling. (Indeed, regular cycles showed a significantly shorter duration of tumescence than did postpartum cycles.)

Of interest was the finding that regular fertile cycles had a significantly shorter tumescent phase than did regular infertile cycles. Similar findings were reported by Dahl et al. (1991). This may suggest that long tumescence in regular cycles is correlated with infertility, since we can assume that females are sexually attractive during both infertile and fertile cycles. In other words, we may assume that the difference between infertile and fertile cycles is purely physiological rather than behavioural. Additional research on sexual behaviour, comparing activity during infertile and fertile cycles, will test the validity of this assumption.

The first tiny swellings of the genitalia, initially involving only the labia, appear when a wild female chimpanzee is around seven years of age (Goodall, 1986). Menarche and full anogenital swellings usually occur $1-3$ years after the first signs of 
swelling in the wild, at approximately 11-13 years of age (Tutin and McGinnis, 1981). First anogenital swellings may appear as early as five years of age in the laboratory (personal observation) with menarche generally occurring by age eight (Keeling and Roberts, 1972).

Under captive conditions, the period of adolescent infertility lasts 4-17 months, averaging just under one year (Young and Yerkes, 1943). Tutin and McGinnis (1981) reported that, for four adolescent females living in Gombe National Park (Tanzania), the phase of infertility lasted 13-34 months. Similarly, Nishida et al. (1990) estimated that adolescent infertility lasted approximately 35 months (2.9 years) in the chimpanzee population of Mahale National Park (Tanzania).

Laboratory chimpanzees are capable of conceiving at eight years of age (Martin, 1981), delivering young at age nine (Wallis, 1982). However, in at least one exceptional case, a captive female chimpanzee conceived at age seven and delivered at age eight (Wallis, unpublished data). The first parturition is estimated to occur at 12-14 years of age in Bossou, Guinea (Sugiyama, 1994). Nishida et al. (1990) reported an average 14.6 years at Mahale and Tutin and McGinnis (1981) gave an estimate of 13-15 years for Gombe.

The present study used data for young females of known age and reproductive events of known timing. The results compare favourably to the estimates provided in earlier field reports. The wide range of results in the study reported here suggests a need to investigate factors influencing delayed puberty versus enhanced reproductive function.

All studies of gestation in chimpanzees suggest an approximate duration of eight lunar months. Some reports have relied on exact figures from timed matings and others use estimated figures by designating the last day of maximal anogenital swelling in the last normal cycle as the day of conception. Average values and ranges from captive study are: 236 days, (Yerkes and Elder, 1936); 228 days (Nissen and Yerkes, 1943); and 231.5 days (range $=192-260 ; n=40 ;$ Wallis and Lemmon, 1986). Under natural conditions, gestation duration was estimated at 224.3 days, using the last day of maximal swelling as the day of conception ( $n=16$, Wallis and Goodall, 1993).

Twin births are rare in chimpanzees. Less than $5 \%$ of pregnancies result in twin births (Peacock and Rogers, 1959) and, as in humans, they typically have a short gestation. The first reported twin birth in captivity was after only 210 days of gestation (Yerkes, 1934) and at least one twin birth occurred after only 192 days (Wallis and Lemmon, 1986). In the wild, the only twin birth of properly estimated gestation was of 206 days (this study).

The gestation duration data used in the present study included a larger data set than that used by Wallis and Goodall (1993), but similar results were obtained. Of importance is the wide range in duration of gestation; the difference between the minimum and maximum figures found in this study (27 days) is greater than the duration of the shortest fertile reproductive cycle (26 days).

A study of 11 captive chimpanzees indicated postpartum amenorrhoea lasted an average of only 28 months (Courtenay, 1987), which is much shorter than that seen in the wild. Postpartum amenorrhoea lasted an average of 42 months (range 11-81 months) at Gombe (Tutin, 1980) and 43 months (range 30-51 months) at Mahale (Hiraiwa-Hasegawa et al.,
1984). The present study resulted in a similar figure for postpartum amenorrhoea after the infant lived (46 months or 3.86 years).

After infant death, females typically resume menstrual cycles very quickly. van Lawick-Goodall (1969) reported that three females exhibited anogenital swellings within a month of their infants' death; the average postpartum amenorrhoea after infant death in the present study was 35 days. Tutin and McGinnis (1981) found that conception occurred within 4-8 months after infant death $(n=5)$. One of their subjects became pregnant during the first postpartum cycle, but the median number of cycles exhibited between parturition and the next conception was 3.6 ( $n=12$, range $=1-11$ cycles). At Mahale, females resumed cycles after an average of 62.6 days, giving birth at an average of 14.4 months, after infant death (Nishida et al., 1990).

The present study examined 26 cases, determining the actual number of days between the onset of postpartum cycles and the probable day of conception. The results confirmed earlier reports that conception typically occurs sooner if the previous infant died than if the infant lived. As yet, no detailed investigation has identified factors that may expedite or delay the timing of conception, thus explaining the wide variability seen in both categories of the analysis.

The average interbirth interval reported in this study $(5.15$ years; $n=11$ ) closely resembles that provided in other reports (early Gombe estimate, Tanzania: mean $=5$ years, 10 months, $n=8$, Tutin and McGinnis, 1981; Mahale, Tanzania: median $=6$ years, Nishida et al., 1990; Bossou, Guinea: 5.1 years, Sugiyama, 1994). Wrangham et al. (1996) report that in the Kanyawara community of the Kibale Forest (Uganda), interbirth interval is estimated to average 7.2 years for 11 subjects. Wrangham suggests that this finding may be related to periods of fruit scarcity in the area. In nearby Ngogo, much more fruit is available and, based on the size differences apparent in siblings travelling with adult females, Wrangham suspects that the interbirth interval at Ngogo is substantially shorter than at Kanyawara, perhaps around 4-5 years (Wrangham, personal communication). Continued work by Kibale researchers will examine whether there is a nutritional mechanism involved in the reproductive ecology of Ugandan chimpanzees.

Not surprisingly, data from captive chimpanzee populations indicate a much shorter interbirth interval than that seen in the wild. Courtenay (1987) found an average interval of 3.8 years in the Taronga Zoo (Sydney). Bloomsmith (personal communication) reported that a 3.5-4.0 year interbirth interval typically occurs in the University of Texas chimpanzee colony. However, the interval is larger for females that have been housed with the same males for many years; as in humans, familiarity in chimpanzees can lead to sexual disinterest and inactivity.

Tutin and McGinnis (1981) identified 16 pregnancies at Gombe from November 1966 to February 1975. For 14 of these it was possible to establish the location and activities of the females at the time of conception. Seven (50\%) conceptions occurred while the female was assumed to be on consortship, suggesting that more females conceive during consort matings than would be expected. This finding led to an interesting discussion of the importance of female choice and mating 
patterns, yet relied on very little data. In the present study, the incidence of consortship conceptions was re-examined more thoroughly, using a much larger data set.

The results from this study indicated that only $25 \%$ (14 of 56) of the known-month conceptions in 1975-1994 could have occurred during a consortship. This figure varies greatly from the $50 \%$ found in the short study by Tutin and McGinnis (1981) and may indicate that consort pairing is less important in overall reproductive strategy than was previously thought. In fact, preliminary assessment of the consort pairs suggests that this mating pattern may be strongly associated with individual preference. For example, of the 117 implied consortships, $26(22.2 \%)$ included the adult male Evered. Evered was never in the alpha position, although he maintained a high rank throughout most of his adult life. He was very successful at coercing females for consort mating and, on three occasions, Evered escorted two females at the same time. During three cases, the female was already pregnant and on four occasions he consorted with infertile females. However, conception was achieved on at least three of his consortships.

Consort mating may also be more preferable for some females than for others. Of the 14 known consort conceptions, four $(28.6 \%)$ were for one female, Patti, who was a member of $19(16.2 \%)$ of the implied consortships under review. Further investigation of the records may confirm whether there is individual preference in the choice of mating patterns.

Although the results of this study did not provide evidence that more females conceive during consort mating than would be expected (cf. Tutin and McGinnis, 1981), this does not dismiss the importance of female mate choice in consortships. Tutin (1979) noted the selection criteria used by females; consort pairings are associated with care-giving behaviour such as grooming and food sharing, not dominance status. These affiliative behaviours may provide the female with a feeling of safety as consort mating away from the community may place the pair at great risk if encountered by a group of chimpanzees from another community. On one well-documented occasion, consort pair Evered and Candy left the Kasakela community on January 27, 1990, and lived well within the territory of the Mitumba community until 30 April 1990. Unfortunately for Evered, Candy did not conceive while on consortship. Instead, she continued to cycle after the pair returned to the Kasakela community and records indicate that conception occurred on approximately 19 June 1990, with parturition 227 days later. In other words, after investing more than three months on a risk-taking consortship into a neighbouring community, Candy conceived while participating in opportunistic mating in the group. (Records indicate that Evered was observed to copulate with Candy at least once on the estimated day of conception.) The results of this study - along with these anecdotal cases suggest the need for further investigation into the costs and benefits involved in chimpanzee consortships (see Morin, 1993).

This review of reproductive parameters considered the records from all individuals that fit the set criteria for inclusion in each analysis. However, an accurate assessment of the Gombe records must include special mention of one remarkable family and their important contribution to our understanding of the reproductive capacity of free-ranging chimpanzees. For the range of values listed in Table 2 , most of the minimum figures were contributed by one female, Fifi, and her eldest daughter, Fanni.

In the early days of habituation at Gombe, one of Goodall's first subjects was Flo, a then ageing female who appeared to have great reproductive success. When she died at an estimated age of 40 , she had given birth to at least five healthy offspring. Her last period of postpartum amenorrhoea lasted only 3 years and 10 months (Goodall, 1968) and, although the last child died in infancy and the juvenile son preceding it died soon after Flo herself, Flo's other offspring became prominent in Gombe's social and reproductive history. Her second born son, Figan, served as alpha male of the Kasakela community for nearly 9 years, and her eldest son, Faben, was also high ranking in the community, despite being partially paralysed by polio (see Goodall, 1986, for complete details).

Flo's eldest daughter, Fifi, has more than matched the reproductive achievements of her mother. At the time of this writing, she has six living offspring and is pregnant for the seventh time - all at an estimated age of 37. Fifi holds the Gombe record for shortest postpartum amenorrhoea after an infant lived ( 2.41 years), shortest interbirth interval ( 3.26 years), shortest gestation (208 days), and tied for the shortest time from onset of postpartum anogenital swelling to next conception (16 days). As mentioned earlier, there was a negative correlation between interbirth interval and Fifi's age.

Fifi's progeny appear to be as socially and reproductively successful as her mother's. Her eldest son, Freud, is the current reigning alpha male in the community and her second-born son, Frodo, is high-ranking and the largest male on recent record at Gombe (approximately $50 \mathrm{~kg}$, personal observation). The first test for paternity exclusion in the Gombe population conclusively identified Frodo as the father of infant Sherehe (Morin et al., 1993), conceived when Frodo was exactly 14 years of age.

Fifi's third-born offspring, Fanni, has now added to the $F$ Family's record-setting reproductive accomplishments. Fanni set the Gombe record for age at first full anogenital swelling (8.5 years), age at first conception (10.5 years), and became Gombe's youngest mother with first parturition at 11 .I years of age. Fifi's fourth offsping, Flossi, exhibited irregular but full swellings at age 9 and by age 10 transferred to the nearby Mitumba community, where she has become a regular (and sexually active) member of the group.

Fifi and Fanni's histories are unusual in free-ranging conditions; in fact, the F Family's reproductive statistics are similar to those of captive chimpanzees. Early maturation and accelerated rates of reproduction in captive settings are thought to be indicators of better nutrition. It is possible that the F Family's diet has influenced their reproductive success.

Female chimpanzees tend to maintain a preferred 'home' range within their community and it happens that the preferred range of Flo, Fifi and now Fanni has been the central region of the Kasakela territory. This area includes the 'feeding station' birthplace of chimpanzee habituation (via banana provisioning). During recent years, provisioning has been greatly reduced; for the period of 1989-1993, females spent less than 1\% of their total feeding time eating bananas (Wallis, unpublished data). However, before that time, large numbers of bananas were provided at the feeding station and members of the F Family have been the most frequent visitors (Goodall, 1968; long-term 


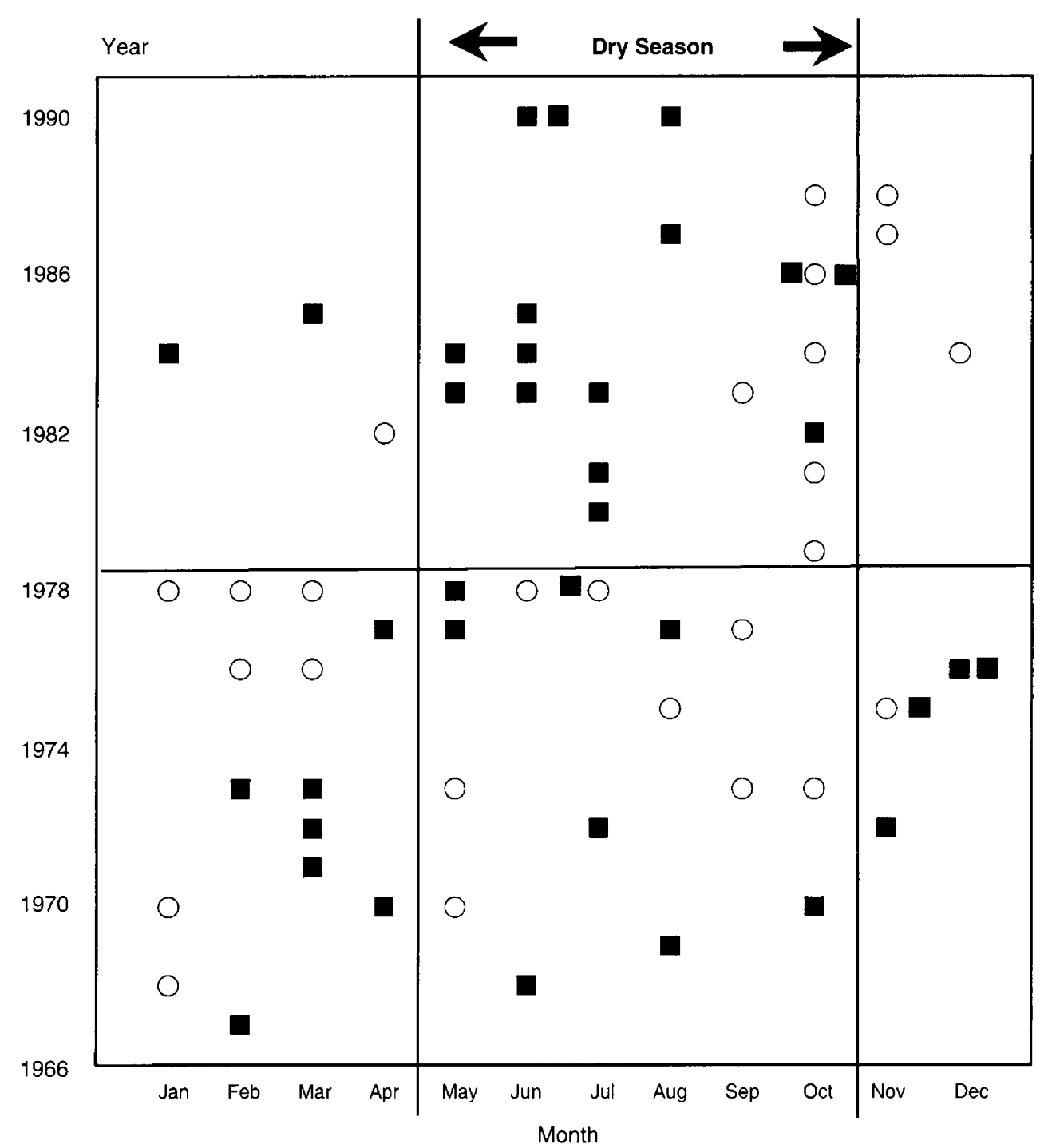

Fig. 1. Known-month conceptions for Gombe chimpanzees, 1967-1990. Dry season is from May to October, inclusive. (O): conception led to miscarriage, stillbirth, or death during infancy; $(\boldsymbol{\square})$ : conception led to live birth and individual lived until at least five years of age. Note the random distribution from 1967 to 1978 compared with the concentration in dry season from 1979 to 1990. In addition, note the pattern of infant mortality $(O)$ concentrated around the months of September to December during the most recent 12 year block.

records, unpublished). It is unclear whether bananas can significantly influence the overall nutrition of chimpanzees or whether other foods may have contributed to the reproductive issues discussed here. It may be that chemical content of the diet (as opposed to nutrition only) may be an important factor. For example, the West African plant Pterocarpus erinaceus contains a substance that may regulate fertility via gonadotrophin release (Benie et al., 1987). A related plant, Pterocarpus tinctorius, is plentiful at Gombe and the chimpanzees eat the fruit, flower, leaf, sap, pith and seed of this species. The flower of the plant is especially important in the diet. It is the thirteenth most important food for males $(2.2 \%$ of their total feeding time) and eighth most important food for females (3.2\% of their feeding time). During March and April, when the flower is most abundant, P. tinctorius flowers become the second most important item on the menu for both males and females $(9.0-13.6 \%$ of the total feeding time). For $1989-1994$, this flower ranked third overall in Fifi's total diet $(5.0 \%$ of her feeding time), first during March ( $13 \%$ of feeding time), and second during April (18\%) (Wallis, unpublished data). On the basis of studies of rodents and humans, an immediate physiological response would not be expected from phytochemical ingestion. Hence, it may be that a female's diet in March or April may influence reproductive activity three or four months later (Wallis, 1992b). As yet, no detailed chemical analysis has been made of $P$. tinctorius, nor has a thorough examination of female diet been conducted for the Gombe females.

Whether the F Family's reproductive accomplishments are due to phytochemicals in the diet, enhanced nutrition, social dominance, 'good genes', or a combination of factors, these data are a reminder that it is important to consider individual differences and potential environmental influences when 
discussing reproduction in chimpanzees. A more thorough examination of nutritional content of the Gombe diet and a detailed assessment of female feeding patterns may provide answers to this question of chimpanzee reproductive ecology.

Complete details of seasonal influence on reproduction in the Gombe chimpanzees are given in Wallis (1995). That study indicated that late dry season is an important time for various aspects of reproduction and fertility. Likewise, in the present study, there was a seasonal influence on infertile cycles of multiparous females; they were shortest during the early dry season, longest during the late dry season, with average duration exhibited during the wet season. There were no significant differences in the duration of anogenital swelling by seasonal quarter; the difference was in the phase of quiescence. This finding is difficult to interpret; however, the short duration in early dry season may suggest that some of these cycles were anovulatory (see Young and Yerkes, 1943). If this is the case, there may be a seasonal effect via dietary content.

One of the more unusual findings of the previous study was a seasonal pattern of conceptions, concentrating in the dry season. However, because of variable duration of gestation, there was no corresponding seasonal effect on time of births (see Wallis, 1995, for details). The data form a more striking pattern when the conceptions are assessed by both month and year of occurrence. All known-month conceptions occurring in the Kasakela community from 1967 to 1990 are illustrated (Fig. 1). (Note: more recent conceptions were omitted for this analysis, so that infant survival could be considered). The figure shows that, during the 12 year block of 1967-1978, there was a random distribution of conceptions throughout the year; of the 35 conceptions identified, $16(45.7 \%)$ occurred during the dry season. However, for the following 12 years (1979-1990), the pattern was not random. Because there were fewer adult females in the community at that time, there were only 27 known-month conceptions. Of these, $21 \quad(77.8 \%)$ occurred during the dry season. A chi-squared analysis of the two seasons by the two 12 year blocks indicated a significant seasonal effect (chi-squared $=8.59, P<0.04$ ).

There may be many factors involved in producing such a seasonal pattern of conception. However, none of these factors, is readily apparent. Over the years, there have been a number of events that effected changes in the Kasakela population: a polio epidemic in 1966; pneumonia epidemics in the mid-1960s and mid-1980s; a community split in 1970-1972; and infanticide committed by adult female Passion in 1974-1977. Although these events influenced changes in the population structure at the time, it is unlikely that they would produce such a drastic - and lasting - change in conception patterns.

It may be that variation in diet (brought about by changes in rainfall) may account for the irregular conception pattern. Rainfall patterns in Gombe have been quite variable over the years. Preliminary assessment of available rainfall data suggest a significant increase in annual rainfall between 1976 and 1982 (J. Moore, personal communication); any possible effect of this on conception patterns starting in 1978, however, would necessarily involve a significant phenological delay.

The data also indicate an unusual pattern in infant mortality; conceptions occurring in the late dry season and early wet season typically led to miscarriage or early infant death during the most recent 12 year block (1979-1990). There is no similar trend in the previous 12 years.

In the earlier investigation of seasonal influence, it was suggested that variation in diet may produce the seasonal pattern of anogenital swelling, conception, and timing of other reproductive events seen in Gombe chimpanzees (Wallis, 1995). There may be an indirect influence of diet; because feeding parties are larger in the dry season, females may be stimulated by one another while in close proximity - effectively enhancing reproductive cyclicity such as synchronization of cycles of anogenital swelling (see Wallis, 1985; 1992b, 1994). Or, as suggested above, diet may directly influence maturation and fertility - either via nutritional content or chemical (phytoestrogen) content.

Future study of female chimpanzee feeding ecology combined with laboratory analysis of diet and faecal steroid content - will help to answer questions generated from the present study. The answers require long-term comparative research from a number of field sites across Africa, with special attention to individual differences in subjects and habitat type. Only then may we begin to understand fully environmental influences on reproduction of this highly vulnerable species and its precarious future.

The author wishes to thank the Field Assistants of Gombe Stream Research Centre, who collected the data reviewed for this study, and Jane Goodall, for generously allowing access to these records. She is also grateful to the Tanzania National Parks, the Commission for Science and Technology of Tanzania, and the Serengeti Wildlife Research Institute for their encouragement and permission to conduct long-term study of the Gombe chimpanzees. The manuscript benefitted from discussion with D. R. Lee, A. Chepstow-Lusty, J. Moore, R. A. Nisbett, D. A. Collins, and especially H. Matama. Portions of the data analysis were supported via NSF grant BNS 8908572

\section{References}

Benie T, el Izzi A, Tahiri C, Duval J, and Thieulant ML (1987) Natural substances regulating fertility. Effect of plant extracts in the Ivory Coast pharmacopoeia on the release of LH by hypophyseal cells in culture Comptes Rendus des Seances de la Societe de Biologie et des ses Filiales 181 163-167

Clark G and Birch HG (1948) Observations on the sex skin and sex cycle in the chimpanzee Endocrinology 43 218-2.31

Courtenay J (1987) Post-partum amenorrhoea, birth intervals and reproductive potential in captive chimpanzees Primates 28 543-546

Dahl JF, Nadler RD and Collins DC (1991) Monitoring the ovarian cycles of Pan troglodytes and Pan paniscus: a comparative approach American Journal of Primatology 24 195-209

Elder JH and Yerkes RM (1936) Chimpanzee births in captivity: A typical case history and report of sixteen births Proceedings of the Royal Society of London Series B 120 409-421

Goodall J (1968) The behaviour of free-living chimpanzees in the Gombe Stream Reserve Animal Behaviour Monographs 1 161-311

Goodall J (1986) The Chimpanzees of Gombe: Patterns of Behavior Harvard University Press, Cambridge, MA

Graham CE (1970) Reproductive physiology of the chimpanzee. In The Chimpanzee, Vol 3 pp 183-220 Ed. GH Bourne. Karger, Basel

Graham CE (1981) Menstrual cycle of the great apes In Reproductive Biology of the Great Apes: Comparative and Biochemical Perspectives pp 1-43 Ed. CE Graham. Academic Press, New York

Hasegawa T and Hiraiwa-Hasegawa M (1983) Opportunistic and restrictive matings among wild chimpanzees in the Mahale Mountains, Tanzania Journal of Ethology 1 75-85 
Hiraiwa-Hasegawa M, Hasegawa T and Nishida T (1984) Demographic study of a large-sized unit-group of chimpanzees in the Mahale Mountains, Tanzania: a preliminary report Primates $25401-413$

Jones JM, Sbarra AJ, and Cetrulo CL (1990) Antepartum management of twin gestation Clinical Obstetrics and Gynecology 33 32-41

Keeling ME and Roberts IR (1972) Breeding and reproduction of chimpanzees In The Chimpanzee 5 pp 127-152 Karger, Baltimore

McGinnis PR (1979) Sexual behavior in free-living chimpanzees: Consort relationships. In The Great Apes pp 429-439 Eds DA Hamburg and ER McCown. Benjamin Cummings, Menlo Park, CA

Martin DE (1981) Breeding great apes in captivity. In Reproductive Biology of the Great Apes: Comparative and Biochemical Perspectives pp 343-373 Ed. CE Graham. Academic Press, New York

Morin PA (1993) Reproductive strategies in chimpanzees Yearbook of Physical Anthropology $36 \quad 179-212$

Morin PA, Wallis J, Moore JJ, Chakraborty R, and Woodruff DS (1993) Noninvasive sampling and DNA amplification for paternity exclusion, community structure, and phylogeography in wild chimpanzees Primates $\mathbf{3 4}$ 347-356

Nadler RD, Graham CE, Collins DC and Kling OR (1981) Postpartum amenorrhea and behavior of apes. In Reproductive Biology of the Great Apes: Comparative and Biomedical Perspective pp 69-81 Ed. CE Graham. Academic Press, New York

Nadler RD, Graham CE, Gosselin RE and Collins DC (1985) Serum levels of gonadotropins and gonadal steroids, including testosterone, during the menstrual cycle of the chimpanzee (Pan troglodytes) American Journal of Primatology 9 273-284

Nishida T, Takasaki H and Takahata Y (1990) Demography and reproductive profiles. In The Chimpanzees of the Mahale Mountains: Sexual and Life History Strategies pp 63-97 Ed. T Nishida. University of Tokyo Press, Tokyo

Nissen HW and Yerkes RM (1943) Reproduction in the chimpanzee: report on forty-nine births Anatomical Record 86 567-578

Peacock LJ and Rogers CM (1959) Gestation period and twinning in chimpanzees Science 129959

Steinetz BG, Ducrot C, Randolph C and Mahoney CJ (1992) Determination of the time of ovulation in chimpanzees by measurement of $\mathrm{LH}$, estrone sulfate, and pregnanediol 3a-glucuronide in urine: comparison with serum hormone patterns Journal of Medical Primatology 21 239-245

Sugiyama $Y$ (1994) Age-specific birth rate and lifetime reproductive success of chimpanzees at Bossou, Guinea American Joumal of Primatology 32 311-318

Tinklepaugh OL (1933) Sex cycles and other cyclic phenomena in a chimpanzee during adolescence, maturity, and pregnancy Journal of Morphology $\mathbf{5 4}$ $521-547$

Tutin CEG (1979) Mating patterns and reproductive strategies in a community of wild chimpanzees (Pan troglodytes schweinfurthii) Behavioral Ecology and Sociobiology $629-38$

Tutin CEG (1980) Reproductive behavior of wild chimpanzees in the Gombe National Park, Tanzania Journal of Reproduction and Fertility Supplement $\mathbf{2 8}$ 43-47
Tutin CEG and McGinnis PR (1981) Chimpanzee reproduction in the wild. In Reproductive Biology of the Great Apes: Comparative and Biomedical Perspectives pp 239-264 Ed. CE Graham. Academic Press, New York

van Lawick-Goodall J (1969) Some aspects of reproductive behaviour in a group of wild chimpanzees, Pan troglodytes schweinfurthi, at the Gombe Stream Chimpanzee Reserve, Tanzania, East Africa Journal of Reproduction and Fertility Supplement 6 353-355

Wallis J (1982) Sexual behavior of captive chimpanzees (Pan troglodytes): pregnant versus cycling females American Journal of Primatology 377 88

Wallis J (1985) Synchrony of estrous swelling in captive group-living chimpanzees (Pan troglodytes) International Journal of Primatology 6 335-350

Wallis J (1992a) Chimpanzee genital swelling and its role in the pattern of sociosexual behavior American Journal of Primatology 28 101-113

Wallis J (1992b) Socioenvironmental effects on timing of first postpartum cycles in chimpanzees. In Topics in Primatology, Volume 1: Human Origins pp 119-130 Eds T Nishida, WC McGrew, P Marler, M Pickford and $\mathrm{F}$ de Waal. University of Tokyo Press, Tokyo

Wallis J (1994) Socioenvironmental effects on first full anogenital swellings in adolescent female chimpanzees. In Current Primatology, Volume II: Social Development, Learning, and Behavior pp 25-32 Eds JJ Roeder, B Thierry, JR Anderson, and N Herrenschmidt. University of Louis Pasteur, Strasbourg

Wallis J (1995) Seasonal influence on reproduction in chimpanzees of Gombe National Park International Journal of Primatology 16 435-4.51

Wallis J and Bettinger T (1993) Sexual attractivity in wild chimpanzees: A comparison of cyclic, pregnant, and lactating females with full anogenital swellings American Journal of Primatology 30 353-354 (Abstract)

Wallis J and Goodall J (1993) Genital swelling patterns of pregnant chimpanzees in Gombe National Park American Journal of Primatology 31 89-98

Wallis J and Lemmon WB (1986) Social behavior and genital swelling in pregnant chimpanzees (Pan troglodyfes) American Journal of Primatology 10 $171-183$

Wrangham RW, Chapman CA, Clark-Arcadi AP and Isabirye-Basuta G (1996) Social ecology of Kanyawara chimpanzees: Implications for understanding the costs of great ape groups. In Great Ape Societies pp 45-57 Eds WC McGrew, LF Marchant, and T Nishida. Cambridge University Press, Cambridge

Yerkes RM (1934) Multiple births in anthropoid apes Science 79 430-431

Yerkes RM and Elder JH (1936) The sexual and reproductive cycles of chimpanzees Proceedings of the National Academy of Sciences USA 22 276-283

Yerkes RM and Elder JH (1937) Concerning reproduction in the chimpanzee Yale Journal of Biological Medicine 10 4I-48

Young WC and Yerkes RM (1943) Factors influencing the reproductive cycle in the chimpanzee: The period of adolescent sterility and related problems Endocrinology 3 121-154 